\title{
A Double-lined Spectroscopic Binary in the Open Cluster Trumpler 14
}

\author{
Hugo Levato $^{1}$ and Stella Malaroda ${ }^{2}$ \\ Complejo Astronómico E1 Leoncito, CC 467, 5400 San Juan, Argentina; levato@infovia.com.ar, malaroda@castec.edu.ar \\ NIDIA MORRELL ${ }^{1,3}$ \\ Facultad de Ciencias Astronómicas y Geofísicas de la UNLP, Paseo del Bosque s/n., 1900 La Plata, Argentina; nidia@fcaglp.unlp.edu.ar \\ BEATRIZ García ${ }^{1}$ \\ Centro Regional de Investigaciones Científicas, CRICYT, Casilla de Correo 131, 5500 Mendoza, Argentina; bgarcia@1ab.cricyt.edu.ar \\ AND \\ MónICA Grosso ${ }^{4}$ \\ Complejo Astronómico El Leoncito, CC 467,5400 San Juan, Argentina; mgrosso@castec.edu.ar \\ Received 1999 August 12; accepted 1999 November 17
}

\begin{abstract}
We present the results of a determination of the preliminary orbital elements of the spectroscopic binary FMM 3 in the field of the open cluster Trumpler 14. The spectrum of this binary shows two sets of lines that are roughly similar. The period found was around 5 days $(P=5.0339 \pm 0.0003)$, and the orbit is eccentric $(e=0.51 \pm 0.02)$. These values were adopted from the combined solution for both components. The mass ratio of the system is $0.76 \pm 0.06$.
\end{abstract}

\section{INTRODUCTION}

Establishing the existence of short-period binaries in compact and dense open clusters is important. Dynamical considerations indicate that the compact and dense nature of these clusters is due to the lack of hard binaries. The open cluster Trumpler 14 in the $\eta$ Carinae complex contains a large number of massive O-type stars. It is compact and seems to be a bound cluster. It is important to know if there is a lack of primordial binaries among its members. It may be argued that, in the case of $\operatorname{Tr} 14$, from the three different processes that make a subgroup in an $O B$ association expand, only the dynamical interactions with binaries remain as the possible source of expansion (see Penny et al. 1993).

Two studies, namely, Levato et al. (1991) and Penny et al. (1993), presented discrepant results about the binary nature

\footnotetext{
${ }^{1}$ Member of the Carrera del Investigador Científico del Consejo Nacional de Investigaciones Científicas y Técnicas de la República Argentina.

${ }^{2}$ Member of the Carrera del Investigador Científico de la Comisión de Investigaciones Científicas de la Provincia de Buenos Aires.

${ }^{3}$ Visiting Astronomer, Complejo Astronómico El Leoncito (CASLEO). CASLEO is operated under agreement of the Universidad Nacional de La Plata, the Universidad Nacional de Córdoba, the Universidad Nacional de San Juan, and CONICET.

${ }^{4}$ Member of the Carrera del Técnico, Consejo Nacional de Investigaciones Científicas y Técnicas.
}

of some of the brightest members of the cluster: for seven stars in common, three of them are spectroscopic binaries according to Levato et al. (1991), while the same stars show no clear radial velocity variations in the work of Penny et al. (1993). García et al. (1998) reobserved the stars in Tr 14 in an effort to resolve the discrepancies. They found that the systematic effect pointed out by Penny et al. (1993) affecting Levato et al.'s data exists, but they also found evidence indicative of binarity in some of the stars observed. In particular, two sets of lines seemed to be present in the spectra of star FMM 3, as designated by Feinstein, Marraco, \& Muzzio (1973).

The present paper is a straightforward effort for determining the preliminary orbital parameters for this star.

\section{OBSERVATIONS}

FMM 3 has been classified as B0.5 IV-V (Morrell, García, \& Levato 1988). Its visual magnitude is 10.80 , and the observed $B-V$ is 0.26 as measured by Feinstein et al. (1973). The quoted $V \sin i, 111 \mathrm{~km} \mathrm{~s}^{-1}$, should be taken with caution because of blended spectral lines even at the resolving power used in this paper.

A total of 23 new spectra of FMM 3 were obtained at CASLEO in the blue region of the spectrum using the Jorge Sahade $2.1 \mathrm{~m}$ telescope and the REOSC echelle spectro- 
graph. ${ }^{5}$ The spectra were recorded on a Tek CCD of $1024 \times 1024$ pixels. We used a grating of 400 grooves $\mathrm{mm}^{-1}$ as a cross-disperser. This instrumental configuration gives a 2 pixel resolution of $0.30 \AA$, with a useful wavelength range of $2000 \AA$ (from 3700 to $5700 \AA$ ). We have also obtained the usual series of bias and flat-field frames. The signal-to-noise ratio of the spectra of the program stars is never larger than 70. A Th-Ar lamp provided the wavelength calibration. During the observations, the spectrograph was conveniently rotated to avoid contamination by HD 93128, a nearby companion at less than $3^{\prime \prime}$ separation. The new 23 spectra observed were added to two spectra whose radial velocities were published by García et al. (1998).

To establish the zero-point correction for our radial velocity determinations, we have obtained spectra for a set of standard stars selected from Feckel (1985) and for HR 1996 and HR 2806, which were used as standards by Penny et al. (1993). No corrections were applied to our data.

All the observations were processed and analyzed using $\mathrm{IRAF}^{6}$ routines.

\section{RESULTS}

Heliocentric radial velocities were derived for each observation by measuring the positions of several $\mathrm{H}$ and $\mathrm{He} I$ lines (when not seriously affected by nebular emission), He II $\lambda 4686, \mathrm{Mg}$ II $\lambda 4481$, and $\mathrm{Si}$ IV $\lambda 4088$. Only average radial velocities are presented in Table 1 , but extensive tables showing line-by-line measurements are also available on request. The position of the spectral lines selected for radial velocity measurements were measured using a Gaussian fitting and deblending routines included in the SPLOT task of IRAF.

Rest wavelengths were taken from Bolton \& Rogers (1978), Moore (1923), and Striganov \& Sventistskii (1968). Table 1 shows the average heliocentric radial velocity for each spectrum of FMM 3 jointly with the Julian date, the probable error of the average, and the number of lines included in the average.

We have looked for possible periods of variation of the radial velocity. With this purpose, we used the code written by Morbey (1978). The best period found is $P=5.033$ days. This period and preliminary orbital elements determined graphically were used as input in Bertiau's (Bertiau \& Grobben 1969) code to compute the final period and the remaining orbital elements. The adopted values are listed in

\footnotetext{
${ }^{5}$ On loan from the Institut d'Astrophysique de Liège.

${ }^{6}$ IRAF is distributed by the National Optical Astronomy Observatories, operated by the Association of Universities for Research in Astronomy, Inc., under cooperative agreement with the National Science Foundation.
}

TABLE 1

Heliocentric Radial Velocities of FMM 3 in Trumpler 14

\begin{tabular}{|c|c|c|c|}
\hline $\begin{array}{c}\text { HJD } \\
(+2,400,000)\end{array}$ & $\begin{array}{l}\text { Radial Velocity } \\
\qquad\left(\mathrm{km} \mathrm{s}^{-1}\right)\end{array}$ & Number of Lines & $\begin{array}{l}\text { Probable Error } \\
\qquad\left(\mathrm{km} \mathrm{s}^{-1}\right)\end{array}$ \\
\hline $49,765.719 \ldots \ldots$ & -18.40 & 10 & 3.6 \\
\hline \multirow{2}{*}{$50,110.788 \ldots \ldots$} & $+79.5(\mathrm{P})$ & 12 & 4.0 \\
\hline & $-106.2(\mathrm{~S})$ & 4 & 3.7 \\
\hline $50,111.778 \ldots \ldots$ & +37.6 & 7 & 4.3 \\
\hline $50,444.807 \ldots \ldots$ & +54.1 & 9 & 3.9 \\
\hline \multirow[t]{2}{*}{$50,446.817 \ldots \ldots$} & $-140.0(\mathrm{P})$ & 11 & 8.0 \\
\hline & $+200(\mathrm{~S})$ & 10 & 8.0 \\
\hline \multirow[t]{2}{*}{$50,447.814 \ldots \ldots$} & +72.6 & 10 & 3.2 \\
\hline & -106 & & \\
\hline $50,480.848 \ldots \ldots$ & $-7.0(\mathrm{P})$ & 16 & 3.9 \\
\hline $50,584.496 \ldots \ldots$ & $\begin{array}{r}+80.0 \\
-121.0\end{array}$ & 12 & 7.5 \\
\hline $50,585.487 \ldots \ldots$ & $+21(\mathrm{P})$ & 17 & 3.2 \\
\hline \multirow[t]{2}{*}{$50,617.482 \ldots \ldots$} & $-117(\mathrm{P})$ & 8 & 3.0 \\
\hline & $+85(\mathrm{~S})$ & 8 & 7.1 \\
\hline $50,618.460 \ldots \ldots$ & $+34(\mathrm{P})$ & 12 & 7.0 \\
\hline $50,821.818 \ldots \ldots$ & $+23(\mathrm{P})$ & 17 & 3.0 \\
\hline \multirow[t]{2}{*}{$50,822.819 \ldots \ldots$} & $-64(\mathrm{P})$ & 16 & 4.7 \\
\hline & $+30(\mathrm{~S})$ & 17 & 3.0 \\
\hline \multirow[t]{2}{*}{$51,207.816 \ldots \ldots$} & $+72.0(\mathrm{P})$ & 8 & 1.3 \\
\hline & $-90(\mathrm{~S})$ & 4 & 1.9 \\
\hline \multirow[t]{2}{*}{$51,208.831 \ldots \ldots$} & $+69(\mathrm{P})$ & 8 & 2.3 \\
\hline & $-87(\mathrm{~S})$ & 3 & 10.0 \\
\hline $51,209.775 \ldots \ldots$ & $+14(\mathrm{P})$ & 11 & 1.7 \\
\hline $51,210.674 \ldots \ldots$ & $-34(\mathrm{P})$ & 8 & 1.0 \\
\hline \multirow[t]{2}{*}{$51,211.662 \ldots \ldots$} & $-131(\mathrm{P})$ & 11 & 1.5 \\
\hline & $+170(\mathrm{~S})$ & 3 & 7.2 \\
\hline $51,215.783 \ldots \ldots$ & $-36(\mathrm{P})$ & 8 & 1.8 \\
\hline $51,216.778 \ldots \ldots$ & $-127(\mathrm{P})$ & 5 & 2.7 \\
\hline \multirow[t]{2}{*}{$51,217.746 \ldots \ldots$} & $+70(\mathrm{P})$ & 11 & 2.1 \\
\hline & $-106(S)$ & 3 & 5.7 \\
\hline \multirow[t]{2}{*}{$51,218.770 \ldots \ldots$} & $+70(\mathrm{P})$ & 8 & 3.3 \\
\hline & $-86(\mathrm{~S})$ & 2 & 6.1 \\
\hline \multirow[t]{2}{*}{$51,275.61 \ldots \ldots$} & $+60.5(\mathrm{P})$ & 14 & 3.9 \\
\hline & $-75.6(\mathrm{~S})$ & 8 & 3 \\
\hline $51,276.570 \ldots \ldots$ & +23 & 13 & 3 \\
\hline \multirow{2}{*}{$51,279.547 \ldots \ldots$} & $+66.9(\mathrm{P})$ & 11 & 5.6 \\
\hline & $-120.5(\mathrm{~S})$ & 4 & 4.2 \\
\hline
\end{tabular}

NoTE. $-(\mathbf{P})=$ primary, $(\mathbf{S})=$ secondary.

Table 2. We have deleted from the computation six spectra with both sets of lines very much blended around phase 0.6 .

Figure 1 shows the computed radial velocity curves for the primary and secondary components with the elements of Table 2, as well as the observations in both cases. The probable error of the fit is $10 \mathrm{~km} \mathrm{~s}^{-1}$.

As a check of the uncertainties of the orbital solution, we have also computed the radial velocity solution for the primary alone. With 20 observations of the primary we have obtained the orbital elements shown in Table 3, which are not very different from those in Table 2, but the probable error of the fit in this case is $5 \mathrm{~km} \mathrm{~s}^{-1}$.

We have located component $\mathrm{A}$ in the theoretical H-R diagram. For this purpose we considered its spectral type as 
TABLE 2

Orbital Elements for Tr 14-3

\begin{tabular}{ccccc}
\hline \hline Element & Primary & \multicolumn{1}{c}{ Error } & Secondary & Error \\
\hline$a \sin i(\mathrm{~km}) \ldots \ldots \ldots \ldots$ & $0.693 \mathrm{E}+07$ & $\pm 0.447 \mathrm{E}+06$ & $0.908 \mathrm{E}+07$ & $\pm 0.491 \mathrm{E}+06$ \\
$e \ldots \ldots \ldots \ldots \ldots \ldots$ & 0.51 & 0.02 & & \\
$K\left(\mathrm{~km} \mathrm{~s} \mathbf{s}^{-1}\right) \ldots \ldots \ldots \ldots$ & 117 & 4 & 153 & 5 \\
$\omega(\mathrm{deg}) \ldots \ldots \ldots \ldots$ & 236 & 5 & & \\
$T_{0}(\mathrm{JD}) \ldots \ldots \ldots \ldots \ldots$ & $2,450,582.94$ & 0.04 & & \\
$P(\mathrm{days}) \ldots \ldots \ldots \ldots$ & 5.0339 & 0.0003 & & \\
$V_{0}\left(\mathrm{~km} \mathrm{~s} \mathrm{~s}^{-1}\right) \ldots \ldots \ldots$ & -5.7 & 3 & & \\
$M_{1} \sin ^{3} i\left(\mathbf{M}_{\odot}\right) \ldots \ldots$ & 3.66 & 0.77 & & \\
$M_{2} \sin ^{3} i\left(M_{\odot}\right) \ldots \ldots$ & 2.79 & 0.69 & & \\
$Q\left(M_{2} / M_{1}\right) \ldots \ldots \ldots \ldots$ & 0.76 & 0.06 & & \\
\hline
\end{tabular}

B0.5 IV-V, and we have used the available photometry, the distance modulus for the cluster, and the bolometric correction derived from the calibration provided by Chlebowski \& Garmany (1991). Comparing the position of component A in the theoretical H-R diagram with the evolutionary tracks computed by Schaller et al. (1992), we find that the mass for the primary is around 20 solar masses while that of component $\mathbf{B}$ is 15 solar masses according to the mass ratio

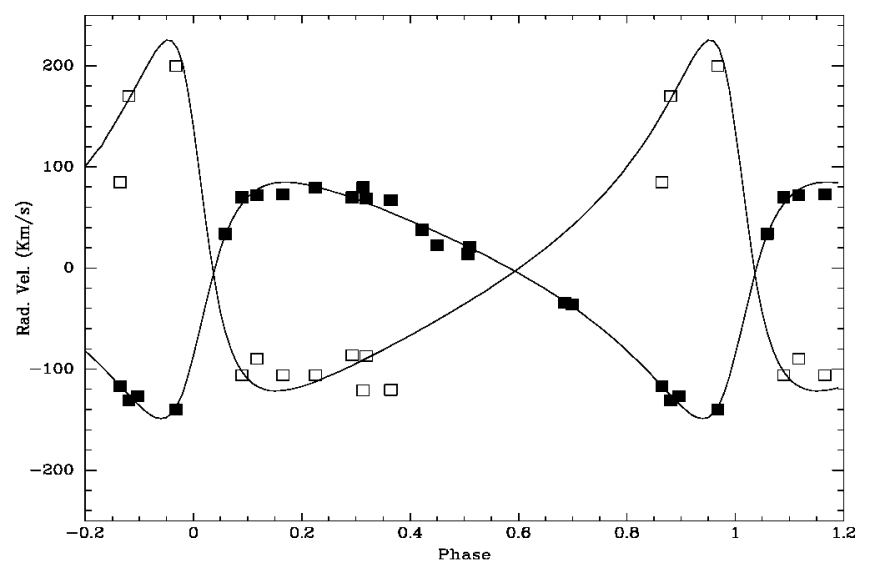

FIG. 1.-Radial velocity curve for FMM 3 in Trumpler 14. Black squares represent the observations of the primary while open squares are for the secondary. The size of the symbols roughly corresponds to the $2 \sigma$ error for the radial velocity measurements.

TABLE 3

\begin{tabular}{ccl}
\multicolumn{3}{c}{ Orbital Elements for TR $14-3 \mathrm{~A}$} \\
\hline \hline Element & Primary & \multicolumn{1}{c}{ Error } \\
\hline$a \sin i(\mathrm{~km}) \ldots \ldots$ & $0.693 \mathrm{E}+07$ & $\pm 0.226 \mathrm{E}+06$ \\
$e \ldots \ldots \ldots \ldots \ldots$. & 0.48 & 0.015 \\
$K\left(\mathrm{~km} \mathrm{~s} \mathbf{s}^{-1}\right) \ldots \ldots$ & 115 & 3 \\
$\omega(\mathrm{deg}) \ldots \ldots \ldots \ldots$ & 239 & 2.5 \\
$T_{0}(\mathrm{JD}) \ldots \ldots \ldots \ldots$ & $2,450,582.91$ & 0.03 \\
$P(\mathrm{days}) \ldots \ldots \ldots$. & 5.0343 & 0.0002 \\
$V_{0}\left(\mathrm{~km} \mathrm{~s} \mathrm{~s}^{-1}\right) \ldots \ldots$ & -3.8 & 1.5 \\
$f(m) \ldots \ldots \ldots \ldots \ldots$ & 0.52 & 0.05 \\
\hline
\end{tabular}

of the system. An inclination of the system of around $35^{\circ}$ results from the above numbers, with no chances of observing eclipses.

\section{DISCUSSION}

One of the points that is not clear yet is the presence of "bound" OB clusters within OB associations, as is the case of Trumpler 14. As Penny et al. (1993) have pointed out, there are only three ways to explain the expansion of the subgroups in an OB association: (a) dispersal of the original gas by the photoionizing flux of the massive stars, $(b)$ mass loss by stellar winds and supernovae, and (c) dynamical exchange involving binaries. In the case of $\operatorname{Tr} 14$ the two first processes can be rejected, and only the dynamical exchange involving binaries remains as responsible for the expansion (see Penny et al. 1993).

Morrell et al. (1988) suggested that the compact appearence of $\operatorname{Tr} 14$ would also be explained if the distance to $\operatorname{Tr} 14$ is larger than the distance to $\operatorname{Tr} 16$, which appears less compact. They suggested two possible distances to $\operatorname{Tr} 14$ : 2.8 and $3.45 \mathrm{kpc}$. Using the results by Morrell et al. (1988), Penny et al. (1993) derived for Trumpler 14 (a) the cluster radius, $1.0-1.2 \mathrm{pc}$; (b) the crossing time for the cluster, (1.11.4) $\times 10^{5} \mathrm{yr}$; and (c) from theoretical evolutionary tracks an age of about $5.5 \times 10^{5} \mathrm{yr}$. On the other hand, Massey \& Johnson (1993) found from extensive CCD photometry that (a) the clusters $\operatorname{Tr} 14$ and $\operatorname{Tr} 16$ are at the same distance and (b) this distance is $3.2 \mathrm{kpc}$ (close to the larger value proposed by Morrell et al. 1988). If their results are correct, then the compactness of $\operatorname{Tr} 14$ could be explained by the anomalously low binary frequency. Hence, it is very important to know this frequency among the members of $\operatorname{Tr} 14$. The results presented in this paper are not in the correct trend in order to support the explanation suggested above for the compact appearance of Trumpler 14. However, detailed observations of the other main-sequence stars in Trumpler 14 are necessary to arrive at a safe conclusion about the level of the binary content of this young cluster. Consider- 
ing the youth of $\operatorname{Tr} 14$, it seems safe to conclude that FMM 3 is a primordial binary. This assumption is also supported by the large eccentricity of the orbit computed in this paper. The barycentric velocity of FMM 3 is around $-5 \mathrm{~km} \mathrm{~s}^{-1}$, not very different than the barycentric velocity for the cluster derived by García et al. (1998) $\left(6 \mathrm{~km} \mathrm{~s}^{-1}\right)$ or by Penny et al. (1993) $\left(4.7 \mathrm{~km} \mathrm{~s}^{-1}\right)$ Finally, it is worth mentioning that the mass ratio for FMM 3 is high compared with that one should expect for a young cluster, if binary forma- tion was due to stellar encounters. See Abt \& Willmarth (1996).

The authors acknowledge use of the CCD and data acquisition system supported under US National Science Foundation grant AST 90-15827 to R. M. Rich. The present investigation was supported by a grant, PIP 4279, from the Consejo Nacional de Investigaciones Científicas y Técnicas (CONICET).

\section{REFERENCES}

Abt, H., \& Willmarth, D. 1996, in ASP Conf. Ser. 90, The Origins, Evolution and Destinies of Binary Stars in Clusters, ed. E. F. Milone \& J. C. Mermilliod (San Francisco: ASP), 105

Bertiau, F. C., \& Grobben, J. 1969, Specola Astron. Vaticana Ricerche Astron., 8, No. 1

Bolton, C. T., \& Rogers, G. L. 1978, ApJ, 222, 234

Chlebowski, T., \& Garmany, C. 1991, ApJ, 368, 241

Feckel, F. 1985, in IAU Colloq. 88, Stellar Radial Velocities, ed. A. G. Davis Philip \& D. Latham (Dordrecht: Reidel), 335

Feinstein, A., Marraco, H. G., \& Muzzio, J. C. 1973, A\&AS, 12, 331

García, B., Malaroda, S., Levato, H., Morrell, N., \& Grosso, M. 1998, PASP, 110, 53
Levato, H., Malaroda, S., García, B., Morrell, N., Solivella, G., \& Grosso, M. 1991, ApJS, 183, 147

Massey, P., \& Johnson, J. 1993, AJ, 105, 980

Moore, C. T. 1923, Princeton Univ. Obs. Contrib., 20, 1

Morbey, C. L. 1978, Publ. Dom. Astrophys. Obs. Victoria, 5, 1

Morrell, N., García, B., \& Levato, H. 1988, PASP, 100, 1431

Penny, L. R., Gies, D. R., Hartkopf, W. I., Mason, B. D., \& Turner, N. H. 1993, PASP, 105, 588

Schaller, G., Schaerer, D., Meynet, G., \& Maeder, A. 1992, A\&AS, 96,268

Striganov, A. R., \& Sventistskii, N. S. 1968, Tables of Spectral Lines of Neutral and Ionized Atoms (New York: IFI/Plenum) 